\title{
Impact of Ping-Pong Events on Connectivity Properties of Node Encounters
}

\author{
Karim Keramat Jahromi, Filipe Meneses, Adriano Moreira \\ Centro Algoritmi \\ University of Minho \\ Guimarães, Portugal \\ karim.karamat@gmail.com, \{filipe.meneses,adriano.moreira\}@algoritmi.uminho.pt
}

\begin{abstract}
Nowadays human mobility modeling is gaining increasing attention from the research community because of its importance in wireless networks, particularly for message delivery in infrastructure-less networks such as Delay Tolerant Networks and Opportunistic Networks. Understanding the role and potential of mobile devices as relaying nodes in message dissemination and delivery depends on the knowledge about encounter patterns among nodes. Data about the usage of Wi-Fi networks can be used to perform an analysis of encounters between pairs of mobile devices, and then be extrapolated for other contexts. One of the main issues in extracting realistic encounters through Wi-Fi traces is the occurrence of ping-pong events that, if ignored, might lead to unrealistic encounter patterns and mislead conclusions. So it is important to deal with this challenge for extracting realistic encounter patterns among mobile nodes. In this paper we evaluate the impact of ping-pong events on main connectivity properties of node encounters such as Contact Time (CT) and Inter Contact Time (ICT) at both individual pairs and aggregate levels. We observed that the impact of ping-pong events at the aggregate level is not pronounced while having significant impact on ICT of some pairs of nodes.
\end{abstract}

Keywords-Mobility modeling, Encounter, Ping-Pong events, Encounters connectivity properties

\section{INTRODUCTION}

The growing popularity of wireless networks, combined with a wide availability of personal wireless devices, provides the basis for communication and access to the Internet. Shortrange wireless devices, such as Wi-Fi enabled devices, are mainly carried by humans thus exhibiting movement patterns that are related to human mobility patterns and behaviors. Such mobility patterns impact the received signal strength and hence impact connectivity models, routing performance, and resource management. It means that movement of mobile nodes impacts the network operation, performance, reliability and even security. The analysis of $\mathrm{Wi}-\mathrm{Fi}$ data traces can provide significant information about human mobility patterns and regularities. This knowledge can be used in many application domains such as urban planning, social science, epidemiology and network communications [1]. Moreover, knowledge about the usage of Wi-Fi networks can be used to perform an analysis of encounters between pairs of devices, and then be extrapolated for other contexts. Mobility and nodal encounters are utilized to deliver messages in intermittently connected Delay Tolerant Networks (DTN) and Opportunistic Networks.
Understanding of nodal encounter patterns is a critical basis for the success of protocols and deployments in DTNs because delivery mechanism depends on nodal encounters. Connectivity properties of human mobility [2] such as Contact Time (CT - the time period while two mobile nodes are in communication range of each other and can communicate directly or indirectly) and Inter Contact Time (ICT - the time elapsed between two consecutive contacts of the same pair of nodes) have a great influence on the performance of message dissemination and delivery in opportunistic networks, especially ICT that can be used to estimate the delay.

A general methodology used in literature $[3,4]$ to study ICT consists in looking at its distribution aggregated over all pairs of nodes (Aggregate ICT), assuming it correctly represents the distributions of individual nodes. However, it has been shown that in heterogeneous networks, when the contact patterns of all pairs are not similar, the aggregated distribution is not representative of the individual pairs' distributions, and looking at that the aggregate distribution can lead to wrong estimation of delay in opportunistic networks [5]. Although there is now a general agreement on the fact that Aggregate ICT (for pedestrians) is distributed according to a power law with a final exponential cut-off $[3,4,5]$ which may lead to infinite expected delay in DTN networks, authors in [5] have shown that pairwise ICTs, which has a great impact on routing, are heterogeneous and follow exponential distributions for a large number of pairs which would guarantee finite expected delay.

The main contribution of this paper is in assessing the impact of ping-pong events on the connectivity properties of node encounters such as CT and ICT distributions. Ping-pong events occur when stations change their association to nearby APs (Access Points) frequently, while these changes aren't the result of actual movement. Although in $[6,7,8,9,10]$ encounters among nodes and some of their distributions have been discussed, and some regularities have been observed, these works ignore some issues in extracting realistic encounters such as ping-pong events in $\mathrm{Wi}-\mathrm{Fi}$ data traces, and ignoring these issues leads to unrealistic encounters traces and mislead conclusions about encounter patterns and even their connectivity properties.

Here we point out the main challenges in extracting realistic encounter events, we discuss ping-pong events in detail as one of the main issues, and propose algorithms for ping-pong events detection and smoothing and also for 
extracting pair node encounters. In this paper we use the terms station, mobile node and also mobile device as being equivalent to each other. The remaining of the paper is organized as follows: in section II we discuss the concept of encounter among mobile devices and also some issues for extracting encounters from Wi-Fi data traces. In section III, conditions for detecting ping-pong events will be considered and then we propose algorithms for detecting and smoothing them. In section IV we suggest another algorithm for extracting encounters and investigate the effect of choosing different values for time thresholds on the total number of encounters. Statistics and trends of the most prominent connectivity properties of encounters, and the impact of ping-pong smoothing on them, are presented in section V. In section VI we discuss related work and finally, in section VII, conclusions and future work are drawn.

\section{PAIR ENCOUNTERS}

An encounter event in the real world means meeting face to face which implies physical proximity among involving objects. The extent of this physical proximity is not always exactly clear and may be different on different scenarios, applications and domains. For instance, in the biological field and in disease spreading, physical proximity or distances between involving objects are short and even can be considered as direct touching, while in wireless networks this proximity (or distances) can be limited by the coverage areas of mobile devices or wireless network infrastructures. Nowadays the majority of short-range wireless devices, such as Wi-Fi devices, are carried by humans. So these devices can be used to observe human mobility behaviors and also to extract physical proximity among human-carried mobile devices in the real world and, as a result, encounter events among humans. Therefore in a communication perspective, an encounter between two mobile devices occurs when they are in communication range of each other or within the coverage area of WLAN infrastructures that devices are associated to (see section IV). Therefore, extracting encounters among people through their portable wireless devices is constrained by the coverage area of devices or infrastructures. In wireless networks, two kinds of encounters are defined: Direct and Indirect. Bluetooth traces track direct encounters between mobile nodes. In this case an encounter occurs when devices come into the radio communication range of each other. WLAN traces record associations between mobile nodes and APs. Even though APs are stationary, they can link mobile nodes that never directly encounter. Therefore, in this case we have indirect encounters, since communication opportunities between nodes are established through APs. Most researchers $[6,9,11,12]$ define an encounter event occurrence in a WLAN when two or more nodes are associated to the same AP during an overlap time interval, and name this as an indirect encounter type. This definition may not always reflect proper and exact realistic physical encounters among nodes due to some challenges. For instance, nodes might be physically close to each other but associated to different APs, or they are out of coverage area of APs, or they are involved in ping-pong events. Next we discuss these challenges with more detail.

In Wi-Fi traces, the location of mobile nodes is approximated by the location of associated APs and its coverage area, but there are several reasons where estimated locations extracted from Wi-Fi traces may not be the real user's location, and this causes some errors in extracting encounters properly. First, nodes aren't necessarily associated with the geographically nearest AP. This behavior may be due to the fact that different APs are being configured with different power levels, access policies or because the signal from the closest AP is being blocked by buildings or other obstacles. Second, different devices have different tendency for changing their associations with APs [13]. Less aggressive devices don't change the associated AP as frequently, thus keeping the current association even while moving around the current AP. The third issue is overlap among the coverage areas of different APs. Although two stations may be in physical proximity of each other, they are associated to different APs. It means that an encounter is occurring in the real world but it is not easy to detect it through the Wi-Fi traces. And finally, ping-pong events $[14,15]$ in which mobile devices change their associations from one AP to another frequently, while they are stationary (when node movement is limited to the coverage area of associated AP, it can be considered as stationary).

In some traces, it has been observed that some nodes have hundreds, sometimes even thousands, handoff events between a few APs. Such scenario is much more likely due to ping-pong events rather than to realistic user mobility. When a mobile device is within the coverage area of multiple APs and due to fluctuations in the received signal strength, nodes often change their associations to the strongest APs. Ping-pong events are especially common where the density of APs in the neighborhood of a node is high and with overlapping coverage areas. The ping-pong events cause many unnecessary handoffs, increase the handoff overhead and reduce the quality of the communication services. On the other hand, ping-pong events lead to unrealistic encounters and mislead conclusions about encounter patterns.

\section{Detecting AND SMOOThING PING-Pong Events}

As mentioned in the previous section, ping-pong events cause errors in extracting real encounters from Wi-Fi traces. So it is necessary to devise a mechanism to eliminate, or at least reduce, their impact in extracting node encounters. One of our goals in this research is to find a solution for detecting pingpong events and smoothing them (reduce high frequency APs switching). Let us consider $t_{D i}$ as the Access Session Time (the time duration that a station is associated to an AP) of a specific station associated to AP $\mathrm{i}$ and $\mathrm{t}_{\mathrm{Ti}}$ as the Transition Time (lag time) between disassociation from the previous AP and association to AP i. In the presence of ping-pong events, a stationary node usually changes its association from one AP to another AP frequently, and this causes a short Access Session Time and, as a result, a short encounter with other stations. But these events are not themselves evidence of a ping-pong event. In addition to short Access Session Time and high rate of change in APs associations, time continuity in APs changing should be considered. Therefore, the following conditions must be satisfied for a ping-pong event to be detected:

- Frequent changes in APs association; 

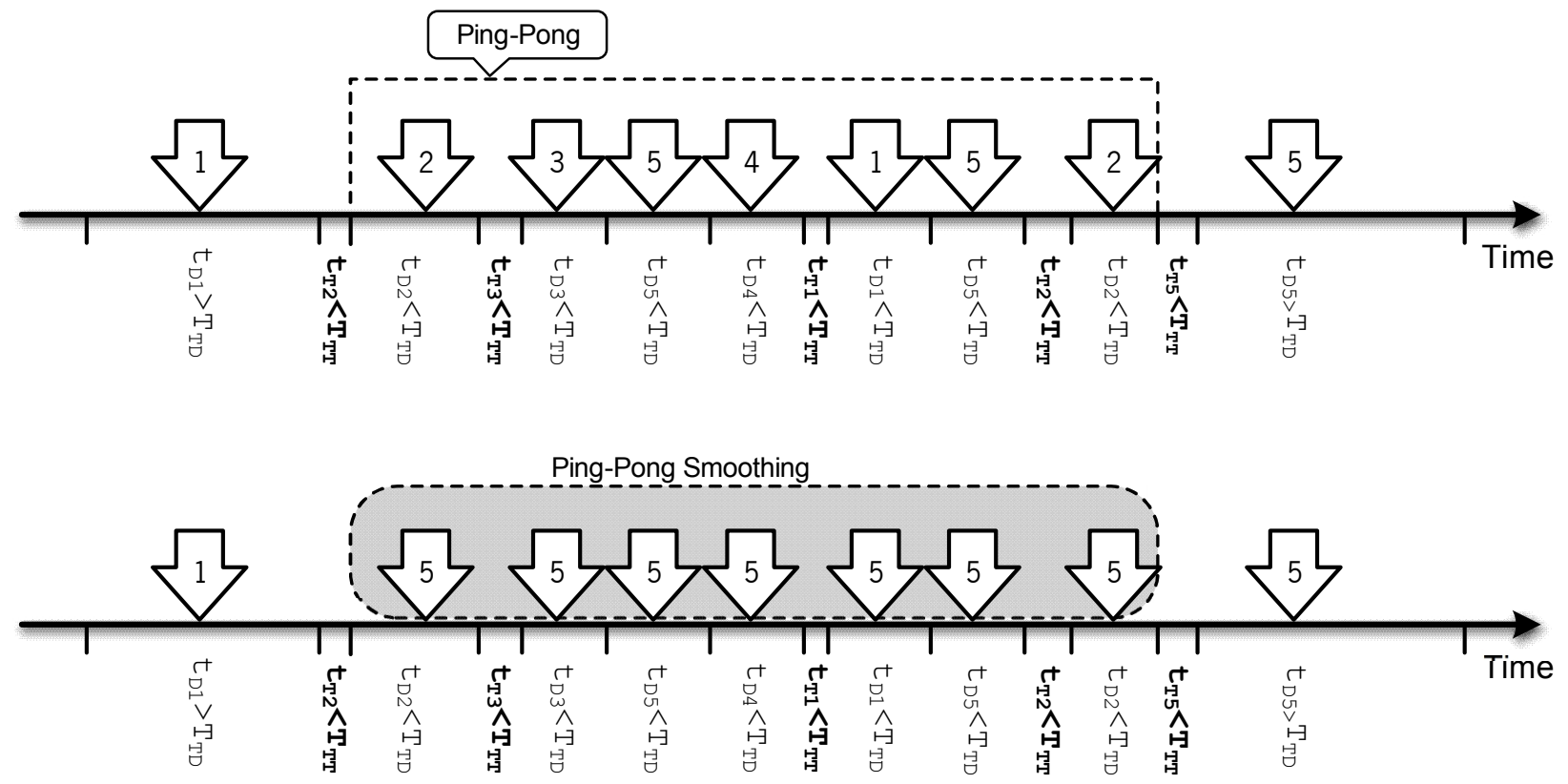

Fig. 1. a) (top) Detecting ping-pong events b) (bottom) Smoothing ping-pong events.

- Continuity in time, meaning that Transition Time between disassociation from previous AP and association to current AP and also Transition Time between changing association from current AP to next AP should be short $\left(\mathrm{T}_{\mathrm{TT}}\right.$ as Transition Time Threshold is considered);

- Short Access Session Time $\left(T_{T D}\right.$ as Access Session Time Threshold should be specified).

The expression "frequent" in the first condition means that more than one change in AP association should occur during $\mathrm{T}_{\mathrm{TD}}+2 \mathrm{~T}_{\mathrm{TT}}$. Chosen values for the above mentioned time thresholds have direct impact on the number of detected pingpong events and on smoothing them. We discuss this matter in section IV. Fig. 1a) displays an example of the occurrence of ping-pong events for a station during a specified time interval, where the arrow represents the station association to an AP. Within this time interval the Access Session Times and Transition Times of the station are less than the defined thresholds, $\mathrm{T}_{\mathrm{TD}}$ and $\mathrm{T}_{\mathrm{TT}}$, respectively, and also there are frequent changes in APs association. A ping-pong interval consists of a set of consecutive node associations to APs which have been involved in ping-pong events and surrounded by two non-ping-pong APs (which are APs that aren't involved in ping-pong events) in the beginning and end of ping-pong interval. In Fig.1a), the ping-pong time interval is highlighted by the dashed line, and is surrounded by the associations to APs 1 and 5 that are not involved in the ping-pong event, since Access Session Times of both of them are higher than the Access session Time threshold $\left(t_{D 1}>T_{T D}, t_{D 5}>T_{T D}\right)$. In this example, the ping-pong event begins with the association of station to AP 2 and ends with disassociation again from AP 2.

\section{A. Detecting ping-pong events:}

Each record in the Wi-Fi trace represent the time period a station is associated to a given AP, with the corresponding start and stop time instants. For detecting ping-pong events in the Wi-Fi trace the following steps should be followed:

1: $\quad$ Find all unique stations in the Wi-Fi trace.

2: $\quad$ For each unique station, find all the unique APs. If the number of unique APs is one, proceed to the next station, otherwise:

2.1: Calculate the Transition Times (between current associated $\mathrm{AP}$ and the previous and next associated AP);

2.2: $\quad$ Mark as being part of a ping-pong event all records for which all the following conditions are satisfied:

2.2.1: Access Session Time shorter than $\mathrm{T}_{\mathrm{TD}}$;

2.2.2: Transition Time shorter than $\mathrm{T}_{\mathrm{TT}}$;

2.2.3: Occurrence of more than one $\mathrm{AP}$ changes during $\mathrm{T}_{\mathrm{TD}}+2 \mathrm{~T}_{\mathrm{TT}}$.

The detailed algorithm for detecting ping-pong events is available in [16].

\section{B. Smoothing ping-pong events}

Smoothing ping-pong events means reducing high frequency APs switching and stabilizing station associations to APs. In [11] authors propose mechanism for smoothing pingpong events using a Weighted Moving Average filter according to the physical coordinates of the APs. They estimate the average location of the station by using its registered locations in the past (within a time window). They assumed the physical coordinates of all APs are available in their trace, while our Wi-Fi trace, and also the majority of other Wi-Fi traces, do not contain any information about the physical coordinates of the APs or even the geographical distribution of the APs.

For smoothing ping-pong for each station we replace associated APs which are involved in ping-pong events with a dominant non-ping-pong AP that is one of two non-ping-pong APs that surrounds a ping-pong interval. The dominant AP is the non-ping-pong AP that has longer Access Session Time. In cases where both non-ping-pong APs have same Access Session Time, the one to which the station associates more 
frequently and/or for longer periods during the ping-pong interval should be chosen as the dominant AP. Fig. 1b) illustrates the smoothing ping-pong process with an example where all APs that are involved in ping-pong events have been replaced by dominant non-ping-pong AP 5. AP 5 is the dominant AP instead of AP 1 since Access Session Time of AP 5 is higher than that of AP $1\left(t_{D 5}>t_{D 1}\right)$, and also because the total time duration of station association to AP 5 is higher than to AP 1 within the ping-pong interval. The complete algorithm for smoothing ping-pong events and choosing the dominant AP is described in [16].

\section{EXTRACTING PAIR ENCOUNTERS}

By considering physical proximity as the criteria for occurrence of encounters among mobile nodes, we can assume mobile nodes are in physical proximity of each other (and as result encountering) if they are associated to the same AP during an overlapping time interval. Since the coverage area of each AP is finite, when two or more nodes are associated to same AP they cannot be far from each other more than the distance corresponding to the diameter of the coverage area. In other words, we use the same encounter definition as other authors $[6,9,11]$ for extracting encounters, but only after detecting and smoothing ping-pong events to reduce the number of cases where a change in association of a node to an AP is due to ping-pong events and not due to realistic movement of the device.

\section{A. Procedure for extracting encounters}

We follow the mechanism below for finding encounters between pairs of nodes from the Wi-Fi trace:

1: $\quad$ Find all unique APs.

2: $\quad$ For each unique AP:

2.1: Find all unique stations that associated to that AP;

2.2: $\quad$ For each unique station from step 2.1:

2.2.1: Compute the temporal overlap between each record and all others records associated to the same AP, except for the records involving the same station. Generate a new encounter record whenever a temporal overlap exists.

The algorithm for extracting pair encounters is listed in [16].

\section{B. Merging pair encounters}

By observing extracted encounters, we discovered that there is a significant number of cases where consecutive encounters between the same pair of nodes are separated by a short time gap (Off-line Time). If this gap is shorter than a predefined threshold $\left(T_{o f f}\right)$, the two encounters can be merged together (see Fig. 2).

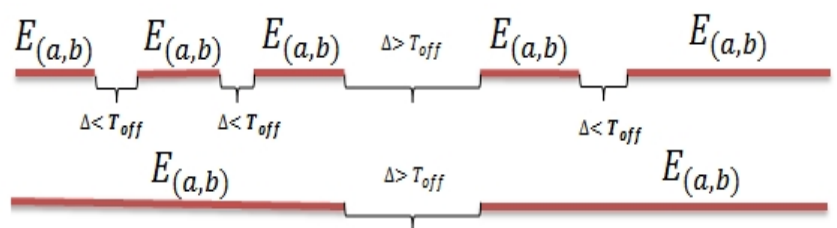

Fig. 2. Merging pair encounters.

Our extracted results show that the total number of encounters is significantly reduced after merging. Also we observed that the number of merged encounters decreases linearly with the value of $T_{o f f}$.

\section{Sensitivity to the values of the Time Thresholds}

The detected encounters are directly influenced by the choice of values for $\mathrm{T}_{\mathrm{TD}}$ and $\mathrm{T}_{\mathrm{TT}}$. In general, a larger $\mathrm{T}_{\mathrm{TD}}$ provides a better guarantee of smoothing the ping-pong events. On the other hand, large $\mathrm{T}_{\mathrm{TD}}$ values also increase the likelihood of accidentally smoothing entries that are part of actual movement. Choosing proper values for these thresholds requires some knowledge about the geographical distribution and coverage areas of the APs, and even movement patterns of the mobile nodes. We investigated the impact of choosing different thresholds for Access Session Time and Transition Time on the number of detected encounters in a data trace collected during 3 months. As shown in Table I, the number of encounters, the number of distinct APs after smoothing pingpong events, and the number of distinct APs in the encounters list depend on the threshold values. These results indicate that by increasing the value of $\mathrm{T}_{\mathrm{TD}}$, the number of encounters increases but the number of distinct A Ps decreases slightly. We also observed changes in the number of ping-pong events. The reason is that by increasing $\mathrm{T}_{\mathrm{TD}}$, the number of stations that are involved in ping-pong increases. As explained before, by replacing APs (in the ping-pong smoothing step) with dominant non-ping-pong APs, which are usually more popular and serve more stations, the number of encounters increases. All the above discussion for $\mathrm{T}_{\mathrm{TD}}$ is applicable to $\mathrm{T}_{\mathrm{TT}}$. We can also observe a significant decrease in the number of encounters after merging with $T_{o f f}=3 \mathrm{~s}$. In order to choose more realistic values for these thresholds we should observe the statistics of Access Session Time and Transition Time. From the analyzed data trace, $\mathrm{T}_{\mathrm{TD}}=5-10 \mathrm{~s}$ and $\mathrm{T}_{\mathrm{TT}}=3-5 \mathrm{~s}$ seem to be adequate values. Although the total number of encounters is quite insensitive to the values of the thresholds, we observed that the number of encounters for some individual pairs of nodes change significantly. We should also consider that the impact of the threshold values is not restricted to the number of encounters but also to the concrete encounters. For instance, if without smoothing ping-pong events, for specific time interval, node $\mathrm{A}$ might be meeting node $\mathrm{B}$ and $\mathrm{C}$, after smoothing node A may be meeting with nodes $\mathrm{D}, \mathrm{K}$ and $\mathrm{J}$. It means that although statistics of aggregate encounters may not change significantly, those associated to specific pairs will be affected drastically. This aspect has a very important impact on routing.

\section{ENCOUNTERS' STATISTICS}

In this section we introduce our Wi-Fi dataset and analyze basic statistics and trends of the detected encounters.

\section{A. The Dataset}

In our experiment we used a Wi-Fi trace extracted from logs of the RADIUS service on two separate campuses of Minho University in two nearby cities in Portugal. Whenever a station (smartphone, tablet or laptop computer) associates or disassociates with an AP, a syslog message is recorded. Each record contains a time stamp in seconds, the MAC addresses of the AP and Station, the Access Session Time in seconds, and the Access Session Status: Start (association), or Stop (disassociation). The analyzed trace was 3 months long, from 1 
Jan 2011 to 31 March 2011. This trace contains references to 1483 distinct APs and 11899 stations. The Wi-Fi data trace does not include any information about the geographic coordinates of the APs or their spatial distributions.

\section{B. Statistics and Trends}

After detecting and smoothing the ping-pong events, and after detecting the encounters, we achieve the results shown in Table I for the first 3 months duration of our trace. By choosing $\mathrm{T}_{\mathrm{TD}}=10 \mathrm{~s}, \mathrm{~T}_{\mathrm{TT}}=5 \mathrm{~s}$ and $T_{\text {off }}=3 \mathrm{~s}$, we observed that 3628 stations (30\%) out of 11899 have been involved in ping-pong events. One interesting result is that encounters have been detected in only $685(46 \%)$ out of the total 1483 APs. This result illustrates the location preference properties of human mobility, confirming that people meet each other in a few popular places [12]. We also observed that 11367 (96\%) out of 11899 Stations get involved in encounters with other stations. The distribution of the number of ping-pong events, normalized by total on-line time per station, is depicted in Fig. 3. This distribution follows a Truncated Power Law trend, which implies that even for stations with long total on-line time, the number of ping-pong events for the majority of stations is low. We also observed that the distribution of ping-pong events per AP follows a Power Law trend.

The distribution of encounter events is important to understand the structure of the relationship among mobile nodes. The understanding of the distribution of encounters helps in assessing the opportunities for message dissemination, prediction of information transmission and message delivery, and estimation of delay. As we mentioned, CT and ICT are two important connectivity properties of human mobility [2] that have prominent role in protocol performance in opportunistic networks. Here we look into the distributions of the Aggregate Inter-Contact Time (AICT), which is aggregated over all pairs of nodes, and also the distribution of ICT for individual pairs of nodes. In most of papers and literatures [3,4], AICT and ACT (Aggregate Contact Time) have been considered as metrics for analyzing the overall behavior of the network and even of individual pairs of nodes.

TABLE I. SENSITIVITY TO THE PARAMETERS VALUES

\begin{tabular}{cccccc}
\hline $\begin{array}{c}\text { Time } \\
\text { Threshold }[\mathbf{s}]\end{array}$ & $\begin{array}{c}\text { Number } \\
\text { of Ping- } \\
\text { Pong } \\
\text { events }\end{array}$ & $\begin{array}{c}\text { Number } \\
\text { of distinct } \\
\mathbf{A P s} \text { in } \\
\mathbf{W i F i} \\
\text { trace }\end{array}$ & $\begin{array}{c}\text { Number of } \\
\text { distinct } \\
\text { inPs } \\
\text { encounters } \\
\text { list }\end{array}$ & $\begin{array}{c}\text { Number of } \\
\text { encounters } / \\
\text { variation } \\
\text { after } \\
\text { smoothing } \\
\mathbf{( \% )}\end{array}$ & $\begin{array}{c}\text { Variation on the } \\
\text { number of } \\
\text { encounters after } \\
\text { merging } \mathbf{( \% )} \\
\left(\mathbf{T}_{\text {off }}=\mathbf{3 s}\right)\end{array}$ \\
\hline $\begin{array}{c}\text { Without } \\
\text { smoothing }\end{array}$ & - & 1483 & 685 & $(7050952)$ & -20.9 \\
\hline $\mathbf{T}_{\mathrm{TD}}=\mathbf{5} ; \mathbf{T}_{\mathrm{TT}}=\mathbf{3}$ & 114831 & 1481 & 685 & +0.45 & $-24,0$ \\
\hline $\mathbf{T}_{\mathrm{TD}}=\mathbf{1 0} ; \mathbf{T}_{\mathrm{TT}}=\mathbf{3}$ & 199361 & 1480 & 685 & +0.90 & -27.7 \\
\hline $\mathbf{T}_{\mathrm{TD}}=\mathbf{1 5} ; \mathbf{T}_{\mathrm{TT}}=\mathbf{3}$ & 258449 & 1480 & 684 & +1.3 & -30.4 \\
\hline $\mathbf{T}_{\mathrm{TD}}=\mathbf{3 0} ; \mathbf{T}_{\mathrm{TT}}=\mathbf{3}$ & 353267 & 1478 & 683 & +1.9 & -34.4 \\
\hline $\mathbf{T}_{\mathrm{TD}}=\mathbf{4 5} ; \mathbf{T}_{\mathrm{TT}}=\mathbf{3}$ & 430137 & 1477 & 681 & +2.4 & -37.8 \\
\hline $\mathbf{T}_{\mathrm{TD}}=\mathbf{3 0} ; \mathbf{T}_{\mathrm{TT}}=\mathbf{5}$ & 388707 & 1477 & 683 & +2.2 & -35.4 \\
\hline $\mathbf{T}_{\mathrm{TD}}=\mathbf{3 0} ; \mathbf{T}_{\mathrm{TT}}=\mathbf{1 0}$ & 441059 & 1474 & 683 & +2.5 & -36.0 \\
\hline $\mathbf{T}_{\mathrm{TD}}=\mathbf{3 0} ; \mathbf{T}_{\mathrm{TT}}=\mathbf{2 0}$ & 483701 & 1473 & 679 & +2.7 & -36.5 \\
\hline $\mathbf{T}_{\mathrm{TD}}=\mathbf{3 0} ; \mathbf{T}_{\mathrm{TT}}=\mathbf{2 5}$ & 497635 & 1473 & 679 & +2.8 & -36.7 \\
\hline $\mathbf{T}_{\mathrm{TD}}=\mathbf{3 0} ; \mathbf{T}_{\mathrm{TT}}=\mathbf{3 0}$ & 506536 & 1472 & 679 & +2.9 & -37.8 \\
\hline
\end{tabular}

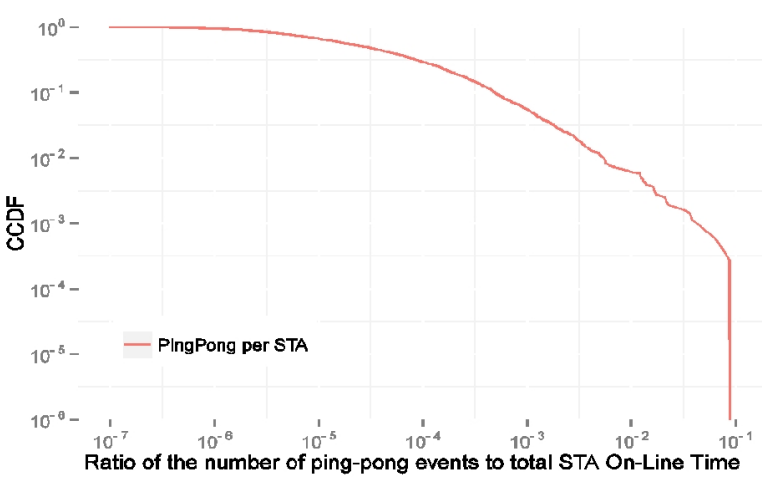

Fig. 3. The distribution the number of ping-pong events normalized by total station On-Line time per station follows a Truncated Power Law.

Fig. 4 shows the distributions of AICT without and with smoothing of ping-pong events, and also after merging encounters, showing that at an aggregated level, the differences are not significant.

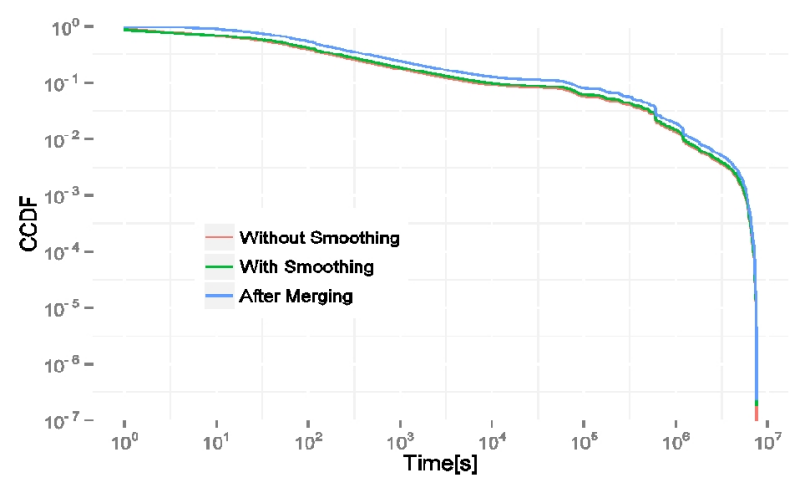

Fig. 4. Comparision of AICT distributions.

The most probable reason for the similarity is that the distribution of the number of ping-pong events per station follows a Power Law trend. Therefore, for the majority of pairs of nodes there isn't a significant difference between ICT before and after ping-pong smoothing. The AICT distributions in all three cases follow a linear decaying Power Law trend until the characteristic time of about 24 hours, and after that truncating exponentially with sharp decaying tail. The average of ICT aggregated over all pairs of nodes for the case with smoothing is around $112 \mathrm{~h}: 34 \mathrm{~min}$ (h: hour ; min: minute). The slopes of the linear decaying part of AICT distribution for the cases with smoothing and after merging, in the time interval from $1 \mathrm{~min}$ to $2 \mathrm{~h}: 38 \mathrm{~min}$, is -0.319 , and in the time interval from $02 \mathrm{~h}: 38$ $\min$ to 20 hours is equal to -0.126 .

These extracted slopes are close to AICT slopes reported in $[4,17]$.

Fig. 5 depicts the distributions of ACT without and with smoothing of ping-pong events, and also after merging encounters, showing that, at an aggregated level, the differences are not significant especially for the cases without and with smoothing where distributions completely overlap. The average of the CT aggregated over all pairs of nodes is around $1 \mathrm{~h}: 33$ min which is much less than the average of the 


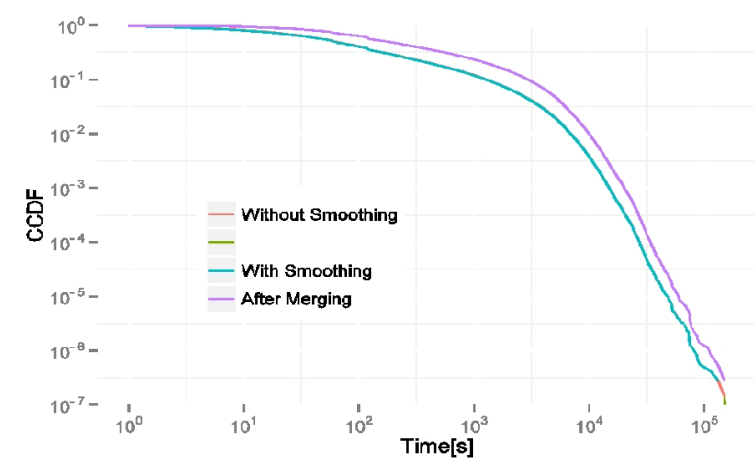

Fig. 5. Comparison of ACT distributions.

ICT aggregated over all pairs of nodes. The ACT distributions (Fig. 5) follow almost a Power Law trend in the time interval from 0 to $30 \mathrm{~s}$ with slope -0.1178 , in time interval from 0.5 to 21 min with slope -0.498 , and after that decreasing exponentially.

The above results indicate that the impact of ping-pong events in the statistics of encounters at aggregate level is not significant. Also, the Truncated Power Law trends in ACT and AICT distributions confirm the heterogeneity in the encounters among mobile nodes. It means that the majority of the encounters have short duration, while a few encounters have long duration. In the case of the AICT, the truncated power law distribution indicates that the majority of pairs of nodes meet each other frequently while some pairs of nodes meet each other very rarely and after long time intervals.

We should note that the distribution of individual pairs' ICT and AICT are not always equivalent. It has been shown that, in heterogeneous networks, when the contact patterns of all pairs are not similar, most of the time the aggregated distribution is not representative of the individual distributions [5]. On the other hand, in many applications, specially routing in opportunistic networks, analyzing the behavior of individual pair of nodes is more important than the behavior of aggregated metrics $[3,4]$. Fig. 6 shows the distribution of ICT for several individual pairs of nodes with different number of encounters during the observed period of 3 months. Different pairs of nodes exhibit different behaviors, illustrating the heterogeneity in the behavior of different pairs and showing that individual ICT distributions are not well represented by the AICT distribution (shown in Fig. 4, which confirms authors' claim in [5]). We also observed a similar behavior for CT distributions of individual pairs of nodes.

Fig. 7 illustrates the distribution of CT and ICT for several pairs of nodes without and with smoothing ping-pong events, and also after merging encounters.

For these particular pairs of nodes, these results show significant differences in the ICT among the three distributions, which in the first two cases (without and with smoothing) highlight the impact of ping-pong smoothing. In almost all pairs of nodes (involved in ping-pong events) we observed that ICT distribution with smoothing is located above the ICT without smoothing. This suggests that at pairwise level ignoring ping-pong events may cause under estimation of delay

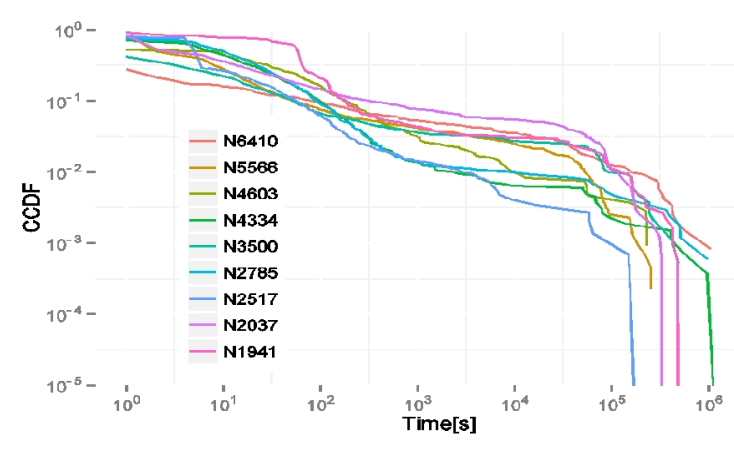

Fig. 6. ICT distribution for a few pairs of nodes with different number of encounter events ( 3 months of data).

for message delivery between pairs of nodes. However, there are other pairs of nodes where these differences are much less pronounced. On the other hand, no significant differences are observed in pairwise CT distributions.

Fig. 8 compares the distribution of AICT and distribution of average of pairwise ICT for all pairs, with smoothing pingpong events. We observe that the AICT distribution is located under the distribution of average of pairwise ICT and there is a significant difference between these two distributions. It implies that the estimated delay through AICT is very under estimated although we know that AICT is not reliable for the estimation of delay for message delivery in opportunistic networks [5].

\section{RELATED WORK}

Mobile devices have become more and more ubiquitous and popular, and analyzing wireless networks formed over these devices is becoming an important research field. Since encounters between nodes provide communication opportunities in DTNs and opportunistic networks, knowledge about node encounters' patterns extracted from collected data traces is important for designing DTNs. These patterns can be uncovered by analyzing trends and statistics of encounters. The majority of works on empirical analysis of WLANs focused on extracting behavior patterns of individual users. Although the understanding of individual behavior is important, it does not reveal how mobile nodes interact with each other and how information can be diffused through relay nodes $[1,15]$. Hsu and Helmy studied the encounters between mobile nodes and introduced the small world approach to explain the encounters relationship graph [6]. They empirically analyzed multiple WLAN traces collected in campus and university environments and extracted some distributions about interactions among mobile nodes and also investigated message diffusion through encounters.

In [7], Wang et al. proposed a generic methodology to extract encounter patterns through an auto persistence function, and they also investigated whether the network formed by periodic encounters has a small world structure to provide communications to a large-scale network. To our knowledge, in these works, the process and issues of extracting encounters have not been considered explicitly. Ignoring these issues, in some cases leads to errors in 


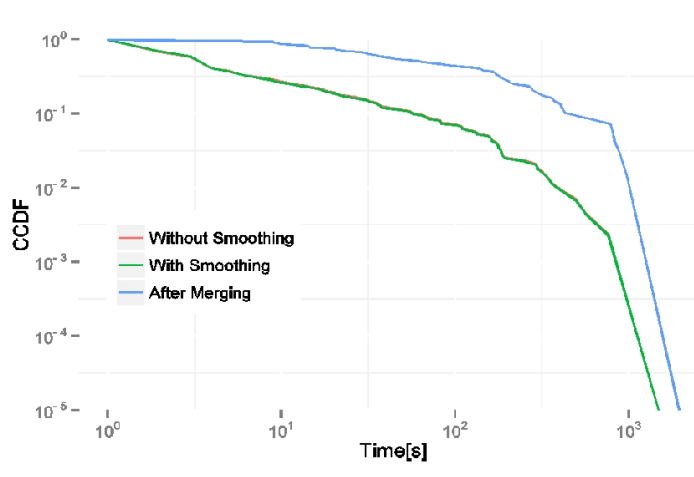

(a1) CT distribution for a specific pair of nodes.

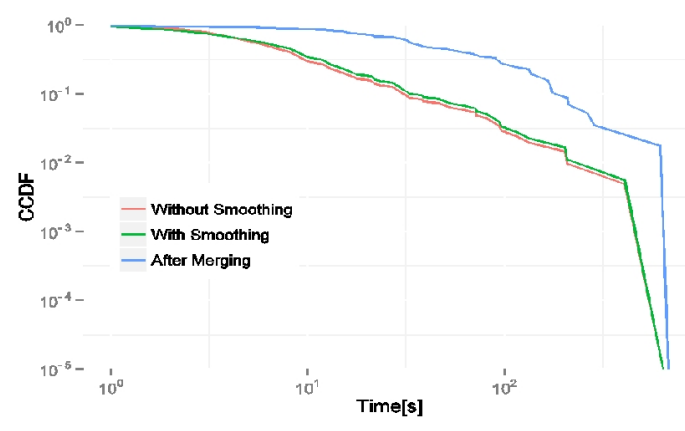

(b1) CT distribution for a specific pair of nodes.

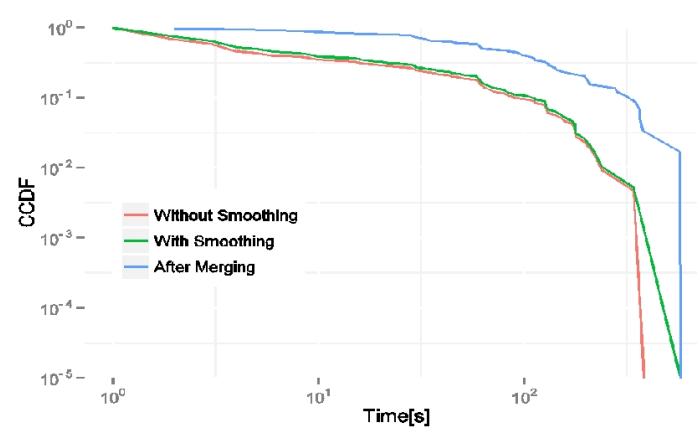

(c1) CT distribution for a specific pair of nodes.

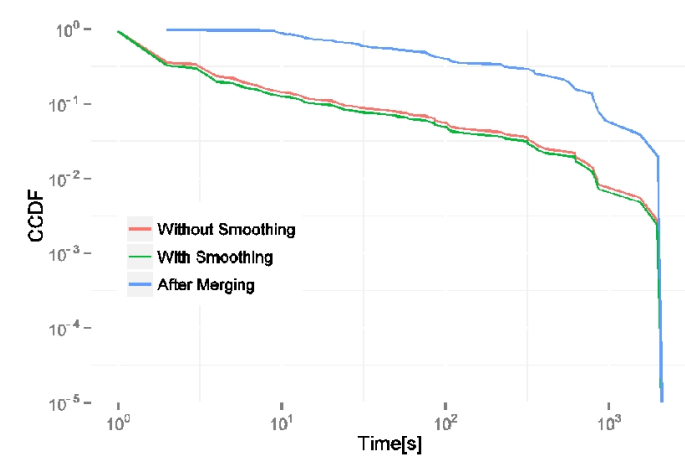

(d1) CT distribution for a specific pair of nodes.

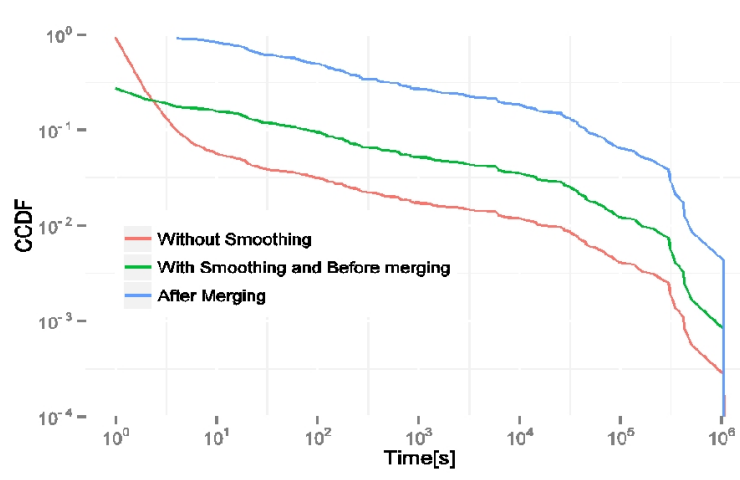

(a2) ICT distribution for a specific pair of nodes.

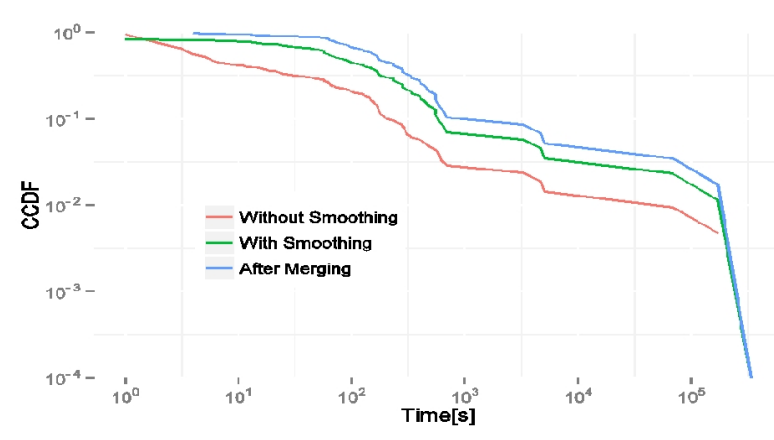

(b2) ICT distribution for a specific pair of nodes.

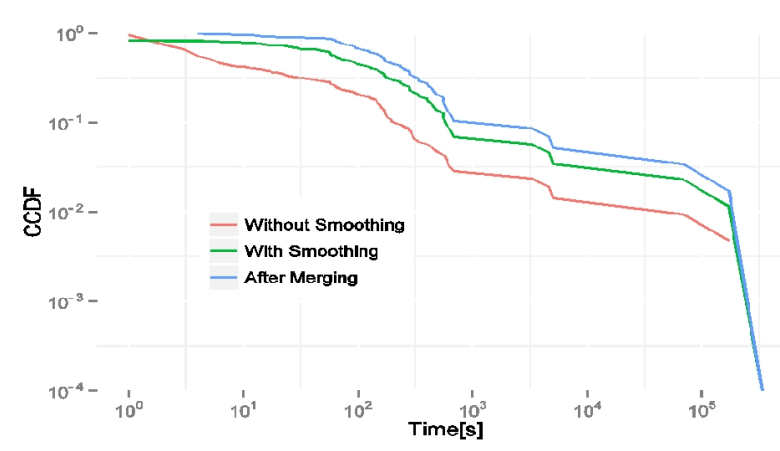

(c2) ICT distribution for a specific pair of nodes.

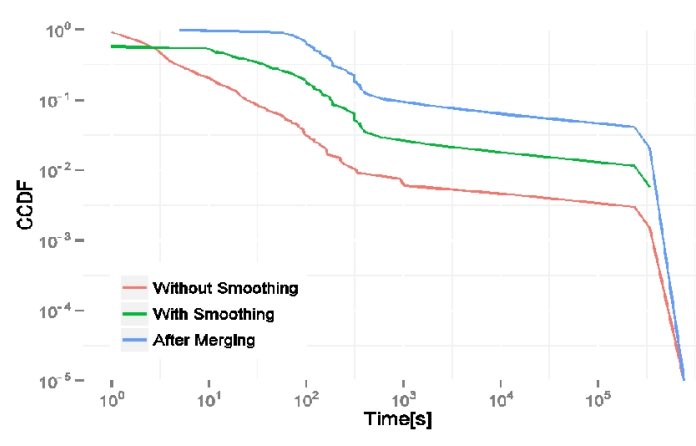

(d2) ICT distribution for a specific pair of nodes.

Fig. 7. Impact of ping-pong events on pairwise CT and ICT distributions 


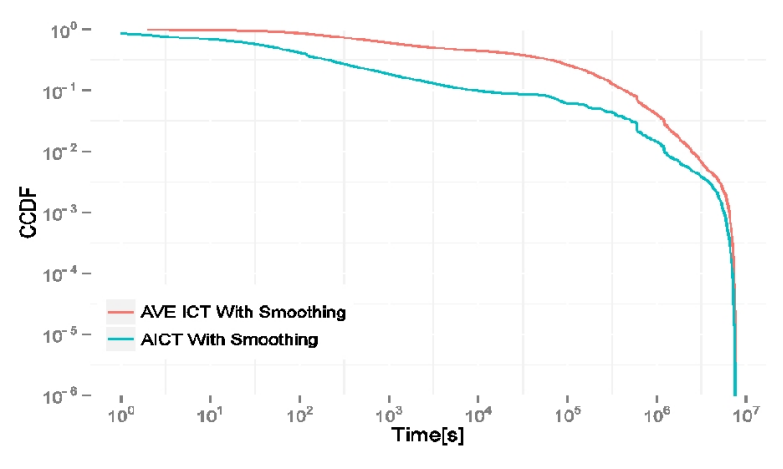

Fig. 8. Comparison between AICT and average ICT.

extracting realistic encounters and, as result, extracted statistics and trends, especially when analyzing individual pair node encounters behaviors, causes significant deviation from realistic results (although in aggregated encounter connectivity distributions differences may not be very significant). In this paper we discussed ping-pong events as one of major issues in extracting real encounters. Yoon et al. [11] proposed the use of a Weighted Moving Average filter for smoothing ping-pong according to the physical coordinates of APs, but their approach is not applicable to our trace since our trace does not contains any information about the physical coordinates of the APs. On the other hand, time thresholds that have been used in their work are, in our perspective, not very realistic. Authors in [17] filter the data with ICT less than 30 minutes to get rid of ping-pong events, which in our perspective may not be a good solution to dealing with this challenge. On the other hand, due to the important role of ICT and CT in estimating the delay and capacity for message delivery in opportunistic networks, there are some studies about distributions of AICT and their relation with pairwise ICT distributions $[3,5,17]$ but by our knowledge, our work is the first one which addresses the impact of ping-pong events on connectivity properties of node encounters.

Although in this paper we just present results for one trace, the extracted aggregated statistics and trends (as we mentioned in Section $\mathrm{V}$, in aggregated cases the impact of ping-pong smoothing is less important) are well aligned with results of other works that have used several data traces from different environments and with longer observation periods.

\section{CONCLUSION AND FUTURE WORK}

In this paper we discussed ping-pong events as one of the major issues in extracting node encounters from Wi-Fi traces. We evaluated the impact of ping-pong events on connectivity properties of human mobility at aggregate and pairwise levels through proposed algorithms for detecting and smoothing ping-pong events from Wi-Fi traces. At aggregate level the impact of ping-pong events on CT and ICT is not significant, but we observed a significant difference in ICT of some pairs of nodes (when both nodes are involved in ping-pong events). On the other hand, in our result we did not observed a significant effect of ping-pong events on individual $\mathrm{CT}$.

As future work we plan to study the issue related to the overlapping in the coverage areas of nearby AP on extracting real encounters and assess their impact on delay in message delivery.

\section{ACKNOWLEDGMENT}

Part of this work has been done within the research project PCSA - Place Characterization from Sensing and Acting, supported by FEDER Funds through the COMPETE and National Funds through FCT Fundação para a Ciência e a Tecnologia (reference EXPL/EEI-SII/0902/2012).

\section{REFERENCES}

[1] M. Gonzalez, C. Hidalgo, A. Barabasi, "Understanding individual human mobility patterns", in Nature 453 (7196)-2008.

[2] Dmytro Karamshuk, Chiara Boldrini, Marco Conti and Andrea Passarella, "Human Mobility Models in Opportunistic Networks", in IEEE Communications Magazine, December 2011.

[3] Karagiannis, T., Le Boudec, J. Y. and Vojnovic, M, “ Power Law and Exponential Decay of Inter-contact Times between Mobile Devices," in IEEE Transactions on Mobile Computing, Vol. 9, 2010.

[4] A Chaintreau, P Hui, J Crowcroft,C Doit "Impact of human mobility on opportunistic forwarding algorithms," in Mobile Computing, IEEE Transactions, 2007

[5] Andrea Passarella and Marco Conti, "Characterizing aggregate intercontact times in heterogeneous opportunistic networks", in Proceedings of the 10th International IFIP TC 6 conference on networking, 2011.

[6] Wei-jen Hsu and Ahmed Helmy, "On Nodal Encounter Patterns in Wireless LAN Traces", in IEEE Transaction on Mobile Computing ,Vol 9, No.11, Nov 2010.

[7] Zhiyu Wang, Mario A. Nascimento and M.H. MacGregor, "On the Analysis of Periodic Mobility Behavior", in the Mobile Data Challenge 2012 (by Nokia) Workshop; 2012; Newcastle, UK.

[8] Jani Kurhinen, Vesa Korhonen, Mikko Vapa, "Modeling Mobile Encounter Networks", in the 17th Annual IEEE International Symposium on Personal Mobile Radio Communications 2006.

[9] Sungwook Moon, Ahmed Helmy, "Understanding periodicity and regularity of nodal encounters in mobile networks:A spectral Analysis", in IEEE Global Telecommunications Conference (Globecom 2010).

[10] Radu Loan Ciobanu and Ciprian Dobre, "Predicting Encounters in opportunistic Networks", Proceedings of the 1st ACM workshop on High performance mobile opportunistic systems, NY, USA, 2012.

[11] Jungkeun Yoon, Brian D. Noble, Mingyan Liu, "Building Realistic Mobility Models from Coarse- Grained Traces", MobiSys '06 Proceedings of the 4th international conference on Mobile systems, applications and services, New York, NY, USA, 2006

[12] Gautam S. Thakur, Udayan Kumar, Ahmed Helmy, Wei-Jen Hsu, "Analysis of Spatio-Temporal Preferences and Encounter Statistics for DTN Performance", 7th International Wireless Communications and Mobile Computing Conference, Istanbul, Turkey, 2011.

[13] Minkyong Kim, David Kotz, Songkuk Kim, "Extracting a mobility model from real user traces", INFOCOM 2006, 25th IEEE Conference on Computer Communications,Spain, 2006.

[14] Kim, Minkyong, Kotz, David, "Modeling users' mobility among WiFi access points", presented at the 2005 workshop on Wireless traffic measurements and modeling, CA, USA, 2005

[15] T. Henderson, D. Kotz, I. Abyzov, "The changing usage of a mature campus-wide wireless network", In International Conference on Mobile Computing and Networking, ACM Press, Sep 2004.

[16] Algorithims for detecting, smoothing and extracting pair encounters are available in http://ubicomp.algoritmi.uminho.pt/?p=1030.

[17] Vania Conan, Jeremie Leguay, Timur Fridman, "Characterizing Pairwise Inter-Contact Patterns in Delay Tolerent Networks," in Proceedings of the 1st international conference on Autonomic computing and communication systems, Belguim, 2007. 